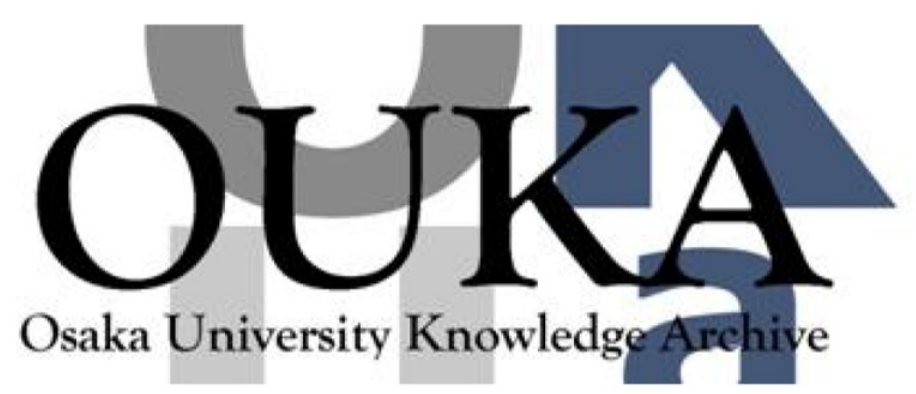

\begin{tabular}{|c|c|}
\hline Title & $\begin{array}{l}\text { Microstitching interferometry for } x-\text { ray } \\
\text { reflective optics }\end{array}$ \\
\hline Author (s) & $\begin{array}{l}\text { Yamauchi, Kazuto; Yamamura, Kazuya; Mimura, } \\
\text { Hidekazu et al. }\end{array}$ \\
\hline Citation & $\begin{array}{l}\text { Review of Scientific Instruments. } 74(5) \text { p.2894- } \\
\text { p. } 2898\end{array}$ \\
\hline Issue Date & $2003-05$ \\
\hline oaire:version & VoR \\
\hline URL & https://hdl. handle. net/11094/85465 \\
\hline rights & $\begin{array}{l}\text { This article may be downloaded for personal use } \\
\text { only. Any other use requires prior permission } \\
\text { of the author and AIP Publishing. This article } \\
\text { appeared in Review of Scientific Instruments } \\
\text { and may be found at } \\
\text { https://doi.org/10.1063/1.1569405. }\end{array}$ \\
\hline Note & \\
\hline
\end{tabular}

Osaka University Knowledge Archive : OUKA

https://ir. Library. osaka-u. ac. jp/

Osaka University 


\section{A|P|P $\mid \begin{aligned} & \text { Review of } \\ & \text { Scientific Instruments }\end{aligned}$}

\section{Microstitching interferometry for $\mathrm{x}$-ray reflective optics}

Kazuto Yamauchi, Kazuya Yamamura, Hidekazu Mimura, Yasuhisa Sano, Akira Saito, Kazumasa Ueno, Katsuyoshi Endo, Alexei Souvorov, Makina Yabashi, Kenji Tamasaku, Tetsuya Ishikawa, and Yuzo Mori

Citation: Review of Scientific Instruments 74, 2894 (2003); doi: 10.1063/1.1569405

View online: http://dx.doi.org/10.1063/1.1569405

View Table of Contents: http://scitation.aip.org/content/aip/journal/rsi/74/5?ver=pdfcov

Published by the AIP Publishing

\section{Articles you may be interested in}

K-edge and mirror filtered $\mathrm{X}$-ray grating interferometers

AIP Conf. Proc. 1466, 229 (2012); 10.1063/1.4742297

High-energy $\mathrm{x}$-ray microbeam with total-reflection mirror optics

Rev. Sci. Instrum. 78, 053713 (2007); 10.1063/1.2736787

Wavefront Metrology for EUV Projection Optics by Soft X-ray interferometry in the NewSUBARU AIP Conf. Proc. 879, 1520 (2007); 10.1063/1.2436354

Relative angle determinable stitching interferometry for hard x-ray reflective optics Rev. Sci. Instrum. 76, 045102 (2005); 10.1063/1.1868472

X-ray wavefront analysis and optics characterization with a grating interferometer Appl. Phys. Lett. 86, 054101 (2005); 10.1063/1.1857066

\section{$\bigoplus$ SHIMADZU Powerful, Multi-functional UV-Vis-NIR and Excellence in Science FTIR Spectrophotometers}

Providing the utmost in sensitivity, accuracy and resolution for a wide array of applications in materials characterization and nanotechnology research

- Photovoltaics - Ceramics

- Polymers

- FPDs

- Thin films

- Coatings

- Paints/inks

- Semiconductors

Click here to learn more
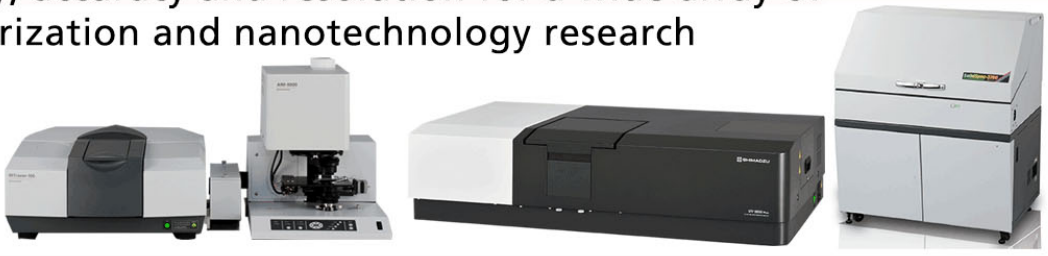


\title{
Microstitching interferometry for $x$-ray reflective optics
}

\author{
Kazuto Yamauchi ${ }^{\text {a) }}$ \\ Department of Precision Science and Technology, Graduate School of Engineering, Osaka University, \\ Yamada-oka 2-1, Suita, Osaka 565-0871, Japan \\ Kazuya Yamamura \\ Research Center for Ultra-Precision Science and Technology, Graduate School of Engineering, \\ Osaka University, Yamada-oka 2-1, Suita, Osaka 565-0871, Japan \\ Hidekazu Mimura, Yasuhisa Sano, Akira Saito, and Kazumasa Ueno \\ Department of Precision Science and Technology, Graduate School of Engineering, Osaka University, \\ Yamada-oka 2-1, Suita, Osaka 565-0871, Japan \\ Katsuyoshi Endo \\ Research Center for Ultra-Precision Science and Technology, Graduate School of Engineering, \\ Osaka University, Yamada-oka 2-1, Suita, Osaka 565-0871, Japan \\ Alexei Souvorov and Makina Yabashi \\ SPring-8/Japan Synchrotron Radiation Research Institute (JASRI), Kouto 1-1-1, Mikazuki, \\ Hyogo 679-5148, Japan \\ Kenji Tamasaku and Tetsuya Ishikawa \\ SPring-8/RIKEN, Kuoto 1-1-1, Mikazuki, Hyogo 679-5148, Japan \\ Yuzo Morib) \\ Department of Precision Science and Technology, Graduate School of Engineering, Osaka University, \\ Yamada-oka 2-1, Suita, Osaka 565-0871, Japan
}

(Received 12 December 2002; accepted 5 February 2003)

\begin{abstract}
A new stitching interferometry based on a microscopic interferometer having peak-to-valley height accuracy of subnanometer order and lateral resolution higher than $20 \mu \mathrm{m}$ was developed to measure surface figures of large-size x-ray mirror optics. Cumulative errors of the stitching angle in a long spatial wavelength range were effectively reduced to be $1 \times 10^{-7}$ rad levels using another interferometer having a large cross section in the optical cavity. Some optical performances of ultraprecise X-ray mirrors, such as submicrofocused beam profile, were wave optically calculated from the measured surface figure profiles and observed at the $1 \mathrm{~km}$ long beamline (BL29XUL) of SPring-8. Observed and wave optically calculated results were in good agreement with a high degree of accuracy. (C) 2003 American Institute of Physics. [DOI: 10.1063/1.1569405]
\end{abstract}

\section{INTRODUCTION}

In third- and coming fourth-generation synchrotron radiation facilities, $\mathrm{x}$-ray beams having laserlike coherency strongly encourage new instrumentation utilizing diffractionlimited focused x-ray beams or coherent x-ray imaging such as nanospectroscopy or phase-contrast tomography. ${ }^{1-3}$ However, such highly coherent $\mathrm{x}$-ray sources demand an unprecedented degree of accuracy in reflective optics to suppress the interference-fringe noises in the reflected $\mathrm{x}$-ray beams. ${ }^{4-6}$ The accuracy criteria to be satisfied in total-reflection mirrors for coherent $\mathrm{x}$-ray sources were wave optically investigated and found to be subnanometer peak-to-valley $(p-v)$ height accuracy over the spatial wavelength range longer than 0.1 $\mathrm{mm} .{ }^{4,6}$ In particular, surface pits and bumps having widths of around $1 \mathrm{~mm}$ were verified to give rise to the interference fringes destructively. Some ultraprecise figure correction

\footnotetext{
${ }^{a}$ Electronic mail: yamauchi@prec.eng.osaka-u.ac.jp

b) Also at: Research Center for Ultra-Precision Science and Technology, Graduate School of Engineering, Osaka University, Yamada-oka 2-1, Suita, Osaka 565-0871, Japan.
}

methods having height accuracy and lateral resolution satisfying the criteria are currently available. ${ }^{7-16}$ Plasma chemical vaporization machining ${ }^{12-16}$ and elastic emission machining $(\mathrm{EEM})^{7-11}$ have demonstrated highly accurate figuring with a spatial resolution close to $0.1 \mathrm{~mm}$. Meanwhile, the measurement of the figure with the required accuracy has become one of the strongest demands. A large variety of highly accurate figure measurement methods having a height accuracy higher than $1 \mathrm{~nm}(p-v)$, such as long trace profilers (LTPs), ${ }^{17}$ laser interferometry, or contact-probe threedimensional figure testing, ${ }^{18,19}$ are currently given as figure testing methods for $\mathrm{x}$-ray reflective optics. However, a lateral resolution as high as $0.1 \mathrm{~mm}$ has not yet been established in figure testers for entire areas of mirror surfaces.

The figure testing technique studied here is a stitching method $^{20,21}$ based on microscopic interferometry. The employed microscopic interferometer head (ZYGO, New view $200 \mathrm{HR}$ ) has a view area of $5.1 \times 3.8 \mathrm{~mm}^{2}$ and a spatial resolution higher than $20 \mu \mathrm{m}$, which satisfies the required lateral resolution in figure measurements of total-reflection mirrors for coherent hard $\mathrm{x}$ rays. Generally, total-reflection mirrors 


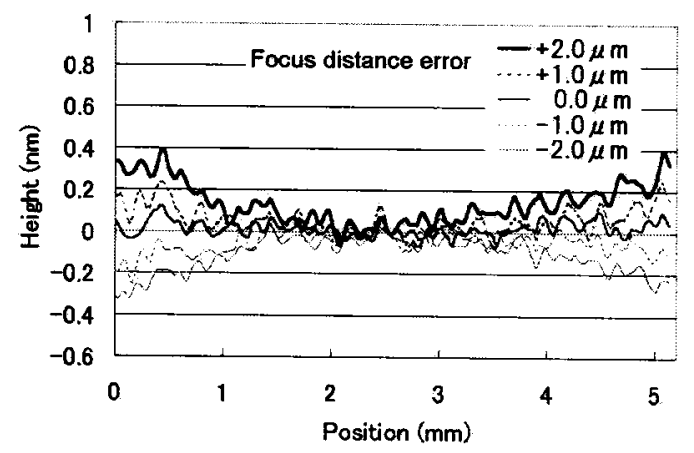

FIG. 1. Measured profiles of an optical flat under various focus distance errors.

have such steep rectangular shapes that the number of stitches in the longitudinal direction often exceeds one hundred. The relative angle between neighboring shots in stitching is actually decided by minimizing the root-mean-square (rms) superposition error in the common area in each shot so that the accuracy of the stitched profile is nearly equal to that realized in each shot so long as the number of stitches is not excessive. However, the angle error $\Delta \theta$ included in each stitching is accumulated and causes problematic error in the long spatial wavelength range when the number of stitches is as large as those in the longitudinal direction of totalreflection hard x-ray mirrors.

In this study, we improved the measurement accuracy in each shot of microscopic interferometry to realize an accuracy of $1 \times 10^{-7} \mathrm{rad}$ in neighboring stitches by developing a posture control system of the optical head both to keep the focal distance constant and to maintain a null fringe setup throughout measuring over the entire mirror surface. In addition, we proposed, in order to remove angle-error integration in long-range stitching, a novel compensation method using another interferometer which does not have sufficient lateral resolution but can measure a large-area profile in one shot.

In this measurement system, which was named microstitching interferometer (MSI), a subnanometer $(p-v)$ height accuracy and a lateral resolution higher than $20 \mu \mathrm{m}$ was realized and confirmed by evaluating the mutual agreement between the optical performances of hard x-ray mirrors actually measured at the $1 \mathrm{~km}$ long beamline (BL29XUL) of SPring- $8^{1}$ and wave optically predicted from the measured figure profiles.

\section{MICROSCOPIC INTERFEROMETRY FOR LARGE- AREA STITCHING}

The focus distance error in microscopic interferometry using imaging optics seriously affects the measurement accuracy because it generates spherically distributed wave front phase errors $\Delta \varphi$, which correspond to the height error $\Delta z$ through the relation $\Delta z=\Delta \varphi \lambda / 2$, where $\lambda$ is light wavelength. ${ }^{21}$ Typical error profiles having a spherical shape obtained by profile measurements of an optical flat under various focus distance errors are shown in Fig. 1. Maximum measurement errors in the slope, which appear at the periphery of view areas and directly affect the stitching accuracy

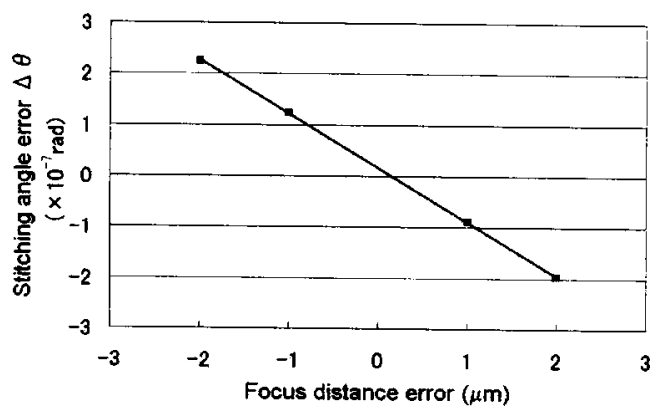

FIG. 2. Relationship between the focus distance error and stitching angle error $\Delta \theta$ estimated by differentiating the profiles shown in Fig. 1.

between neighboring shots, were evaluated by differentiating the error profiles in Fig. 1 and plotted against focus distance errors in Fig. 2. The relation shows that a focus distance error smaller than $1 \mu \mathrm{m}$ is necessary to realize angle accuracy higher than $1 \times 10^{-7} \mathrm{rad}$ in stitching. In the present interferometer, a feedback system, the configuration of which is schematically shown in Fig. 3, is installed to maintain a constant focus distance during microscopic imaging of the entire surface of the mirror. Mirrors are placed on the $X Y$ scanning stage with three piezoelectric displacement devices (PI, P-733) which can enable two-dimensional tilting and elevating, and is aligned automatically to maintain the focus distance within one dark or bright fringe interval $(\approx 0.15$ $\mu \mathrm{m})$ and to keep the null fringe setup using interference fringe patterns simultaneously monitored by an image processing system. The null fringe setup is not essential to the measurement accuracy but is effective for realizing highly accurate measurement under environmental instabilities such as the vibration of the instrument or index fluctuations caused by airflow turbulence in the optical path. Using such a feedback system, reproducibility higher than $1 \times 10^{-7} \mathrm{rad}$ of the slope at the periphery of the view area was obtained in independently measured profiles of the same surface.

On the other hand, system error, including imperfection of the reference plate and lens aberrations, which corresponds to absolute accuracy in figure testing, was compensated for to be less than $1 \mathrm{~nm}(p-v)$ and $0.1 \mathrm{~nm}(\mathrm{rms})$ by a well-known statistical compensation method of averaging 20 sets of profiles measured at the different points on the optical flat having a sufficiently random profile in roughness. ${ }^{22}$ The

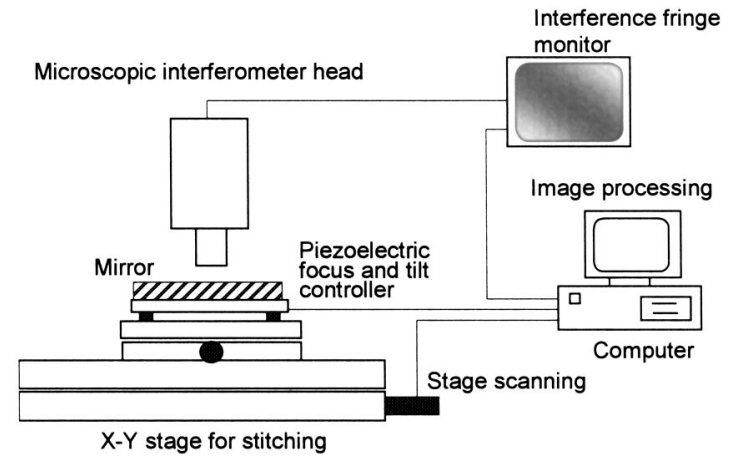

FIG. 3. Schematic of microscopic interferometer in which the mirror tilt and $z$ position can be adjusted to maintain the null-fringe setup and constant focus distance by simultaneously monitoring the interference fringe image. 


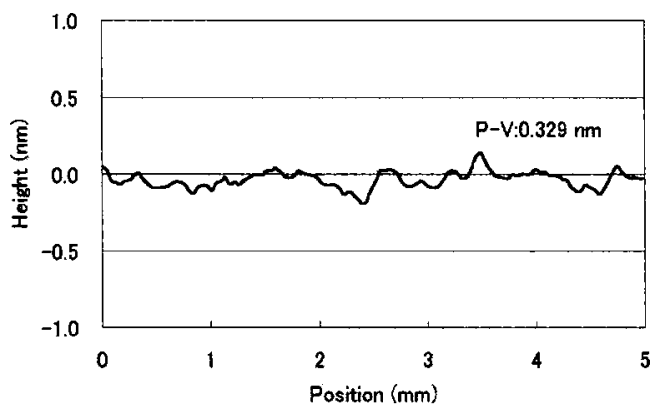

FIG. 4. Typical difference profile between two independently measured system errors.

absolute reliability of the system error can be confirmed at every measurement by evaluating the difference profile between the two sets of system errors obtained independently. A typical difference profile is shown in Fig. 4, and the difference is seen to be small enough to confirm the high absolute reliability of the obtained system error profiles.

\section{STITCHING ERROR COMPENSATION}

The accuracy of the stitched figure profile in the long spatial wavelength range is still doubtful because the angle error $\Delta \theta$ has some possibility of being integrated to an unacceptable value even when each shot of microscopic imaging has a sufficient slope accuracy such as $1 \times 10^{-7} \mathrm{rad}$. The ambiguity can be removed by employing another interferometer which has a large cross section in the optical cavity and has sufficient accuracy in the long spatial wavelength range. For this purpose, a Fizeau interferometer (ZYGO, GPI XPHR) with a large reference plate of $200 \mathrm{~mm}$ diameter, of which the absolute profiles along several lines near the centerline were proven to have the accuracy of $1 \mathrm{~nm}(p-v)$ using a three-flat test ${ }^{23,24}$ generally adopted to measure the absolute shape of a nearly flat surface. The lateral resolution $(0.4 \mathrm{~mm})$, which corresponds to the pixel size of the image detector and zooming parameter, is small enough compared to the view area size of the microscopic interferometer that the stitching angle error $\Delta \theta$ at each common area can be estimated using a sufficient number of height data in each shot. However, the Fizeau interferometer does not have sufficient reproducibility of the measured profiles in the relatively short spatial wavelength range, such as shorter than 20 $\mathrm{mm}$, because of environmental instability, against which the large optical cavity has some disadvantages concerning measurement accuracy. The difference profile between the independently measured profiles of a cylindrical surface is shown in Fig. 5. Height differences are distributed around a few nanometers $(p-v)$ in the relatively short wavelength range such as $1-20 \mathrm{~mm}$ with a notable relationship with the interference fringe pattern. In contrast, a slope accuracy of 1 $\times 10^{-7} \mathrm{rad}$ and height accuracy of around $1 \mathrm{~nm}(p-v)$ are obtained in the spatial wavelength range longer than $20 \mathrm{~mm}$, as understood from the low-pass-filtered profile having a cutoff length of $20 \mathrm{~mm}$ also shown in Fig. 5. Based on the results, the stitching angle error $\Delta \theta$ can be estimated from the difference between the simply stitched profile of the microscopic interferometer images and appropriately low-pass-

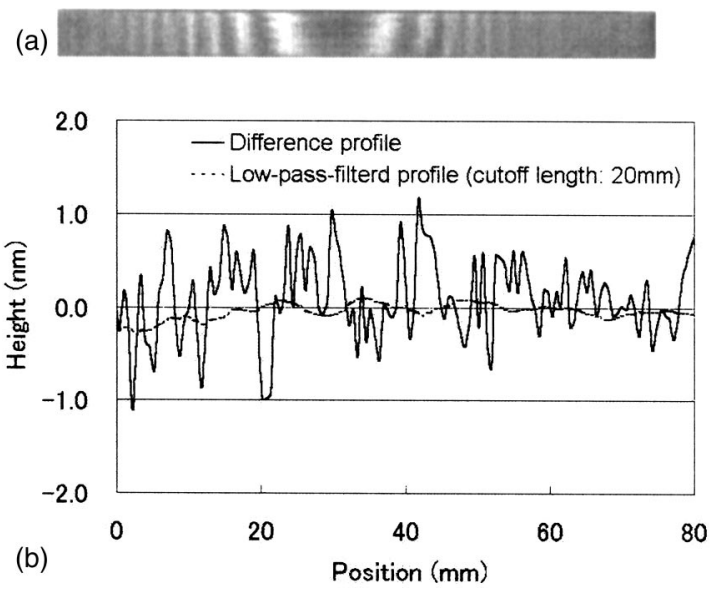

FIG. 5. (a) Interference fringe image of the measured area. (b) Difference profile between independently measured surface profiles of a cylindrical surface. Bold and fine lines are raw and $20 \mathrm{~mm}$ cutoff low-pass-filtered data.

filtered profile obtained using the Fizeau interferometer. An evaluation width, which corresponds to the cutoff length in the low-pass filtering of the Fizeau interferometer image and in which the angle error of each microscopic interferometer shot is evaluated and corrected to fit the Fizeau interferometer image, is employed as an adjustable parameter to achieve the maximum accuracy in the stitched profile considering the following relationships. When the evaluation width is too narrow, the estimated stitching angle error $\Delta \theta$ at each superposed area is affected by the measurement error of the Fizeau interferometer in the short spatial wavelength range. Under such a condition, $\Delta \theta$ is estimated to be a value larger than $1 \times 10^{-7} \mathrm{rad}$ that is a guaranteed value in each microscopic interferometer imaging as just mentioned. In contrast, when the evaluation width is too wide, the estimated $\Delta \theta$ becomes smaller than the guaranteed value. However, in this case, the figure profile obtained using the Fizeau interferometer is not utilized effectively because some parts of the real surface profile included in the measured data are removed by the too strong low-pass filtering. In Fig. 6, estimated $\Delta \theta$ s of the surface shown in Fig. 5 are plotted for every common area in stitching. The evaluation widths compared here are $10 \mathrm{~mm}, 15 \mathrm{~mm}, 20 \mathrm{~mm}$, and $25 \mathrm{~mm}$. The maximum value of the estimated $\Delta \theta$ is seen to decrease with greater evaluation width and becomes $1 \times 10^{-7} \mathrm{rad}$ at the evaluation width of $25 \mathrm{~mm}$ in this case. They continue to

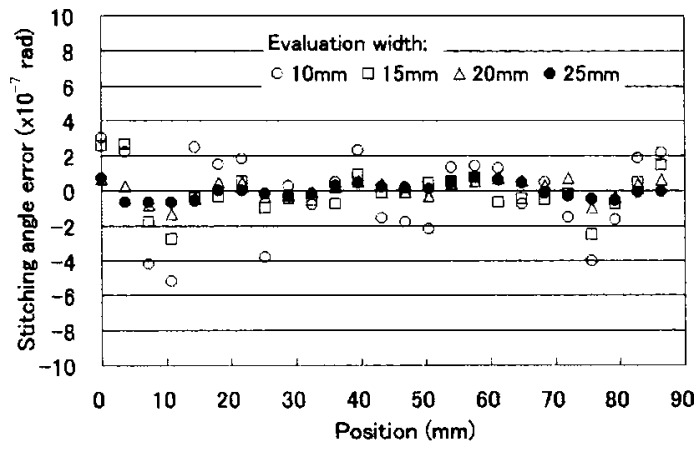

FIG. 6. Relationship between evaluation width and stitching angle errors $\Delta \theta$ at each common area. 


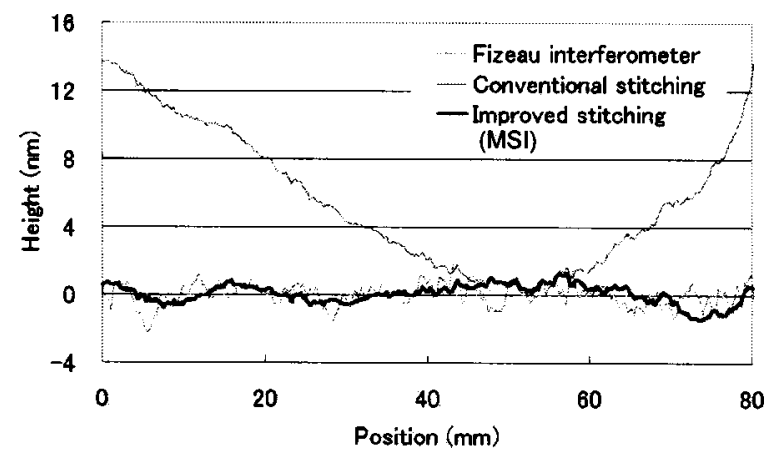

FIG. 7. Figure error profiles measured using microscopic, Fizeau, and combined interferometers. The target figure in this case was an ellipse having maximum depth of about $3 \mu \mathrm{m}$.

become smaller with a further increase of evaluation widths. Then, the optimum width in this case can be determined to be $25 \mathrm{~mm}$. In other words, the expected slope accuracy in this figure measurement is preset to be $1 \times 10^{-7} \mathrm{rad}$ over the spatial wavelength range longer than the lateral resolution of the microscopic interferometer, unless the height fluctuations of $0.1 \mathrm{~mm}(p-v)$ order randomly appearing in the interferometer are a dominant factor against the obtainable accuracy in the relatively high spatial wavelength range. Such procedures to determine the best evaluation widths are done separately in suitably sectioned areas in the case that the interference fringe pattern density in the Fizeau interferometer image is widely changed on the area to be measured.

Surface profiles measured by the conventional stitching, Fizeau, and improved stitching interferometers are compared in Fig. 7. The dotted line in Fig. 7 is the figure profile directly obtained using the large-area Fizeau interferometer. The fine and bold solid lines in Fig. 7 are those obtained using the present interferometer with a simple stitching and improved stitching methods, respectively. Measurement errors in the short spatial wavelength range of the Fizeau interferometer are removed in the optimally compensated profile obtained by the improved method of the MSI. In addition, those in the long spatial range in the simply stitched profile of the microscopic interferometer are also removed.

\section{ABSOLUTE ACCURACY AND DISCUSSION}

We here show some examples of estimating the absolute accuracy of the MSI. Figure 8 shows the figure profiles of the flat mirror before and after numerically controlled flattening by EEM, together with a profile precalculated in the figure correction procedures in which the stitching method was utilized to obtain the surface figure profiles. A photograph of the x-ray beam reflected by the figure-collected mirror, indicating that an intensity flatness better than $5 \%$ is realized, is also presented in Fig. 8. The interference fringes in totally reflected coherent hard x-ray beams are investigated in detail and verified to fade upon removing nanometer-order pits and bumps in the spatial wavelength range from submillimeter to a few decades of millimeter. The findings, shown in Fig. 8, i.e., that the thickness to be removed from the premachined surface was measured by the stitching method, the corrected surface has a profile very

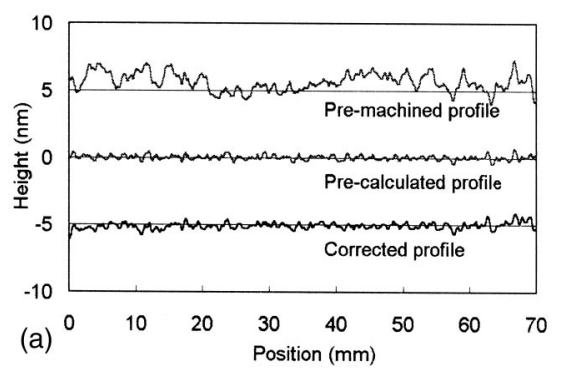

X-ray image reflected at figure corrected area ( $15 \%$ of the total area on each side is not figure-corrected)

(b) X-ray image reflected at noncorrected area

FIG. 8. (a) Premachined, precalculated and actually corrected surface profiles of total reflection flat mirror. (b) Intensity images of the $\mathrm{x}$ ray reflected at figure-corrected and noncorrected areas on the flat mirror. Images are obtained at the $1 \mathrm{~km}$ long beamline of SPring- 8 by an $\mathrm{X}$-ray camera (HAMAMATSU, C5333). X-ray wavelength and glancing angle are 0.06 $\mathrm{nm}$ and $1.2 \mathrm{mrad}$, and the distance between the mirror and the X-ray camera was $166 \mathrm{~mm}$.

similar to the predicted one, and the intensity flatness of the $\mathrm{X}$-ray beam reflected by the mirror surface is better than $5 \%$, show sufficient reproducibility and absolute accuracy of the MSI for it to be used as a figure testing method of totalreflection flat mirrors for coherent hard $\mathrm{x}$ rays.

Figure 9(a) shows the x-ray beam profile focused by an elliptically figured mirror together with the wave optically calculated beam profile from the surface figure profile shown in Fig. 9(b) which was measured by the MSI. The focused beam profile was actually observed at the $1 \mathrm{~km}$ long beamline of SPring-8. ${ }^{25}$ The subpeak on the left-hand side was analyzed to originate in a period of sinusoidlike height error near the center of the mirror, which is clearly seen in the figure profile shown in Fig. 9(b). The amplitude and spatial period of the sinusoidlike curve are about $5 \mathrm{~nm}$ and $40 \mathrm{~mm}$, respectively. Good agreement between the measured and reproduced beam profiles is obtained despite the influence of slope errors as small as $1 \times 10^{-7} \mathrm{rad}$. This means that the figure measurement system has sufficient absolute accuracy to fabricate and evaluate ultraprecise focusing mirrors for hard $\mathrm{x}$ rays of aspherical shape.

The most important points to realize high measurement accuracies in MSIs are how to guarantee the slope accuracy in microscopic interferometer images and the reliability of the employed large-area profiler in a relatively low spatial frequency range. Concerning the latter, Fizeau interferometer employed in this study has a $200 \mathrm{~mm}$ diameter optical cavity, which is rather small for large $\mathrm{x}$-ray optics having longitudinal sizes more than several $100 \mathrm{~mm}$. However, in the conceptual viewpoint, many kinds of other large-area profilers are applicable in principle; larger cavity interferometers, glancing angle interferometers, LTPs, and three-dimensional contact-probe profilers are suitable examples so far as the required height accuracy is guaranteed in the long spatial wavelength range. 

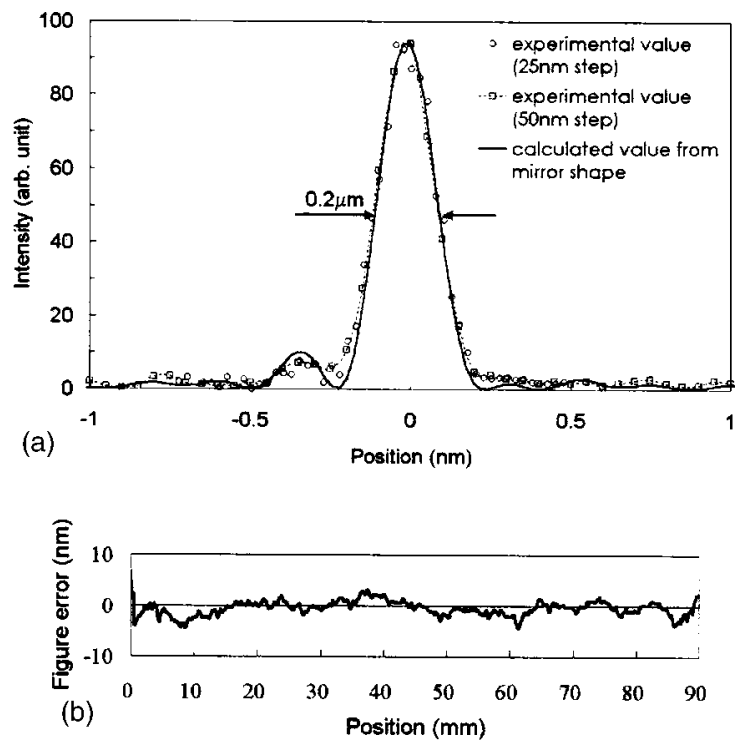

FIG. 9. (a) Measured and reproduced intensity profiles of the focused x-ray beam. Measured profile was obtained at the $1 \mathrm{~km}$ long beamline of SPring- 8 by a wire scanning method with a gold wire having $0.2 \mathrm{~mm}$ diameter. X-ray wavelength was $0.08 \mathrm{~nm}$. The mirror surface profile measured by the MSI was taken into account in the reproduced beam profile. (b) Surface figure error profile measured by the MSI. Length and breadth of the ellipse employed as a surface profile of the mirror are $1000.3 \mathrm{~m}$ and $48.5 \mathrm{~mm}$, and effective mirror size in the longitudinal direction and focus distance are 90 and $300 \mathrm{~mm}$

\section{ACKNOWLEDGMENT}

This work was supported by a Grant-in-Aid for COE Research (No. 08CE2004) from the Ministry of Education, Sports, Culture, Science, and Technology of Japan.

${ }^{1}$ T. Ishikawa, K. Tamasaku, M. Yabashi, S. Goto, Y. Tanaka, H. Yamazaki, K. Takeshita, H. Kimura, H. Ohashi, T. Matsushita, and T. Ohata, Proc. SPIE 4154, 1 (2001).

${ }^{2}$ B. C. Larson, W. Young, G. E. Ice, J. D. Budai, and J. Z. Tischler, Nature (London) 415, 887 (2002)

${ }^{3}$ C. Martin, G. Eeckhaut, A. Mahendrasingam, D. J. Blundell, W. Fuller, R. J. Oldman, S. J. Bingham, T. Dieing, and C. Riekel, J. Synchrotron Radiat. 7, 245 (2000).
${ }^{4}$ Y. Mori, K. Yamauchi, K. Yamamura, H. Mimura, A. Saito, H. Kishimoto, Y. Sekito, M. Kanaoka, A. Souvorov, M. Yabashi, K. Tamasaku, and T. Ishikawa, Proc. SPIE 4501, 30 (2001).

${ }^{5}$ A. Souvorov, M. Yabashi, K. Tamasaku, T. Ishikawa, Y. Mori, K. Yamauchi, K. Yamamura, and A. Saito, J. Synchrotron Radiat. 9, 223 (2002).

${ }^{6}$ K. Yamauchi, K. Yamamura, H. Mimura, Y. Sano, A. Saito, M. Kanaoka, A. Souvorov, M. Yabashi, K. Tamasaku, T. Ishikawa, and Y. Mori (unpublished).

${ }^{7}$ K. B. Becker, in Precision Science and Technology for Perfect Surfaces, edited by Y. Furukawa, Y. Mori, and T. Kataoka (Japan Society for Precision Engineering, Tokyo, 1999), p. 51.

${ }^{8}$ Y. Mori, K. Yamauchi, and K. Endo, Precis. Eng. 9, 123 (1987).

${ }^{9}$ Y. Mori, K. Yamauchi, and K. Endo, Precis. Eng. 10, 24 (1988).

${ }^{10}$ Y. Mori, K. Yamauchi, K. Endo, T. Ide, H. Toyota, K. Nishizawa, and M. Hasegawa, J. Vac. Sci. Technol. A 8, 621 (1990).

${ }^{11}$ K. Yamauchi, K. Hirose, H. Goto, K. Sugiyama, K. Inagaki, K. Yamamura, Y. Sano, and Y. Mori, Comput. Mater. Sci. 14, 232 (1999).

${ }^{12}$ Y. Mori, K. Yamauchi, K. Sugiyama, K. Inagaki, S. Shimada, J. Uchikoshi, H. Mimura, T. Imai, and K. Kanemura, in Precision Science and Technology for Perfect Surfaces, edited by Y. Furukawa, Y. Mori, and T. Kataoka (Japan Society for Precision Engineering, Tokyo, 1999), p. 207.

${ }^{13}$ Y. Mori, K. Yamamura, and Y. Sano, Rev. Sci. Instrum. 71, 4627 (2000).

${ }^{14}$ Y. Mori, K. Yamauchi, K. Yamamura, and Y. Sano, Rev. Sci. Instrum. 71, 4620 (2000)

${ }^{15}$ H. Takino, N. Shibata, H. Itoh, T. Kobayashi, H. Tanaka, M. Ebi, K. Yamamura, Y. Sano, and Y. Mori, Jpn. J. Appl. Phys., Part 2 37, L894 (1998).

${ }^{16}$ H. Takino, N. Shibata, H. Itoh, T. Kobayashi, H. Tanaka, M. Ebi, K. Yamamura, Y. Sano, and Y. Mori, Appl. Opt. 37, 5198 (1998).

${ }^{17}$ P. Z. Takacs, Proc. SPIE 749, 59 (1987).

${ }^{18}$ M. Negishi, K. Watanabe, K. Matsusita, T. Kasahara, and K. Hosaka, in Precision Science and Technology for Perfect Surfaces, edited by Y. Furukawa, Y. Mori, and T. Kataoka (Japan Society for Precision Engineering, Tokyo, 1999), p. 354.

${ }^{19}$ H. Shiozawa and Y. Fukutomi, in Precision Science and Technology for Perfect Surfaces, edited by Y. Furukawa, Y. Mori, and T. Kataoka (Japan Society for Precision Engineering, Tokyo, 1999), p. 360.

${ }^{20}$ M. Bray, Proc. SPIE 4451, 375 (2001).

${ }^{21} \mathrm{~S}$. Chakmakjian, J. Biegen, and P. de Groot, Technical Digest of International Workshop on Interferometry (Riken, Japan, 1996), p. 171

${ }^{22}$ MetroPro $^{\mathrm{TM}}$ Reference Guide Version 7. 4. 2 OMP-0347E (Zygo Corporation, 2001), p. 37.

${ }^{23}$ R. A. Smythe, J. A. Soobitsky, and B. E. Truax, Proc. SPIE 816, 95 (1987).

${ }^{24}$ W. Primak, Appl. Opt. 6, 1917 (1967).

${ }^{25}$ K. Yamauchi, K. Yamamura, H. Mimura, Y. Sano, A. Saito, A. Souvorov, M. Yabashi, K. Tamasaku, T. Ishikawa, and Y. Mori, J. Synchrotron Radiat. 9, 313 (2002). 